\title{
Successful long-term use of pioglitazone in Berardinelli-Seip lipodystrophy-associated diabetes
}

\author{
Carolina Chaves ${ }^{1}$, Mariana Chaves ${ }^{2}$, João Anselmo ${ }^{1}$ and Rui César ${ }^{1}$ \\ 'Serviço de Endocrinologia e Nutrição and 2Serviço de Radiologia, Hospital Divino Espírito Santo de Ponta Delgada, \\ Ponta Delgada, Açores, Portugal
}

Correspondence should be addressed to $C$ Chaves

Email

carolina.m.chs@gmail.com

\section{Summary}

Berardinelli-Seip congenital lipodystrophy (BSCL) is a rare autosomal recessive disease, characterized by the absence of subcutaneous adipose tissue, leptin deficiency and severe metabolic complications, such as insulin resistance, diabetes mellitus, and dyslipidemia. The most common mutation occurs in BCSL2 which encodes seipin, a protein involved in adipogenesis. We report a patient with BSCL who was diagnosed with diabetes at 11 years old. He was started on metformin 1000 mg twice daily, which lowered glycated hemoglobin (HbA1c) to less than 7\%. Four months later, HbA1c raised above $7.5 \%$, indicating secondary failure to metformin. Therefore, we added the peroxisome proliferator-activated receptor-gamma (PPARG) agonist, pioglitazone. Since then and for the last 5 years his $\mathrm{HbA1c}$ has been within the normal range. These findings indicate that pioglitazone should be considered as a valid alternative in the treatment of diabetes in BSCL patients. To the best of our knowledge, this is the first specific report of successful long-term treatment with pioglitazone in a patient with BSCL.

\section{Learning points:}

- Berardinelli-Seip congenital lipodystrophy (BSCL) is a recessive genetic disorder associated with severe insulin resistance and early onset diabetes, usually around puberty. Failure of oral antidiabetic medication occurs within the first years of treatment in BSCL patients.

- When failure to achieve metabolic control with metformin occurs, pioglitazone may be a safe option, lowering insulin resistance and improving both the metabolic control and lipodystrophic phenotype.

- Herein we show that pioglitazone can be a safe and efficient alternative in the long-term treatment of BSCL patients with diabetes.

\section{Background}

Berardinelli-Seip congenital lipodystrophy (BSCL) is a rare disease, with an estimated prevalence of 1 in 10 million worldwide and 1:500 000 cases in Portugal (1). It is characterized by the absence of subcutaneous adipose tissue since birth and metabolic alterations such as insulin resistance, diabetes, and dyslipidemia. There are four genes associated with the disease: AGPAT2 (type 1), BSCL2 (type 2), CAV1 (type 3), and PTRF (type 4). The two most frequent types are type 1 ( $40 \%$ of the cases) and type 2 (50\%). The BCSL2 encodes seipin, a protein involved in adipogenesis and lipid droplet formation (1). In vivo, ex vivo and in vitro studies using BSCL2 deficient mice showed that seipin plays a critical role in the homeostasis of adipose tissue and that in its absence patients present severe lipodystrophy with the absence of adipose tissue (2). This lack of adipose tissue leads to leptin deficiency, resulting 
in an inability to store fat in the subcutaneous adipose tissue, which alternatively accumulates in muscles and liver causing hepatosplenomegaly, hypertriglyceridemia and insulin resistance, creating, therefore, a snowball effect (3). Although the diagnosis can be suggested by careful clinical observation, it must always be confirmed by genetic testing (4). Type 2 BSCL patients typically have serious complications diagnosed earlier in life, requiring specific medical therapy. High insulin levels acting through IGF-1 receptors are associated with accelerated bone age, muscular hypertrophy including hypertrophic cardiomyopathy and early pubertal signs. The risk of premature atherosclerosis and cardiac failure is high (5). Therapeutic options are based on symptomatic control of complications. Metformin and pioglitazone have been used to treat partial lipodystrophies, but are not usually useful in patients with generalized lipodystrophies. To the best of our knowledge, this is the first described case report of a patient with BSCL type 2 treated with pioglitazone and metformin for a long period of time (over 5 years), with good metabolic control.

\section{Case presentation}

The 16 years old caucasian boy herein described was born after an uneventful pregnancy from a non-consanguineous couple. Four months after birth, physical examination revealed a child with acromegaloid features, muscular hypertrophy and absence of adipose tissue. His neurologic and psychomotor development appeared normal. At 18 months, he showed liver and spleen enlargement at physical examination and performed an abdominal ultrasound that showed sonographic characteristics of hepatic steatosis and splenomegaly. The laboratory study revealed hypertriglyceridemia of $4.36 \mathrm{mmol} / \mathrm{L}$ (normal 0.39-2.20) and abnormal hepatic function with high transaminase levels. The echocardiogram showed hypertrophy of the ventricular septum. At this time, the presumptive diagnosis of BSCL was made. The patient was kept in a low-fat diet without medication. At 5-year-old, it was noticed acanthosis nigricans in cervical, axillary and periumbilical regions. At 9 years old, he was diagnosed with hypertension. At 11 years old, he was referred to our Pediatric Endocrinology consultation because of fasting hyperglycemia (plasma glucose: $15.8 \mathrm{mmol} / \mathrm{L}$, normal: 3.89-6.11) and high glycated hemoglobin (HbA1c: 9\%, normal: 4-6\%). Glucose tolerance test confirmed diabetes. The patient exhibited severe acanthosis nigricans involving the cervical, axillary and periumbilical areas. Lipodystrophy was obviously evident in the face, trunk and limbs and together with prominent muscular hypertrophy conferred to the patient an athletic appearance (Fig. 1). The HOMA IR index was 5.71 (normal: <2.6), indicating severe insulin resistance. Further analytic evaluation demonstrated normal thyroid function; an increased C-peptide of 1.59 nmol/L (normal: 0.36-1.46); Insulinemia $281.3 \mathrm{pmol} / \mathrm{L}$ (normal: 35.8-71.6) and microalbuminuria of $144.9 \mathrm{mg} /$ day (normal: 1.5-20). The serum leptin was not detectable. The genetic analysis showed homozygosity for insertion of a single adenosine residue in codon 699 , which resulted in premature truncation of seipin. This mutation had been previously reported in BSCL type 2 patients from South Africa of Portuguese ancestry (6)) and was the same mutation of a patient's cousin who lives in Canada.

\section{Treatment}

At 9 years old because of uncontrolled hypertension, the patient started atenolol $50 \mathrm{mg} /$ day, and enalapril $5 \mathrm{mg} /$ day. At 11 years old, he was treated with metformin,

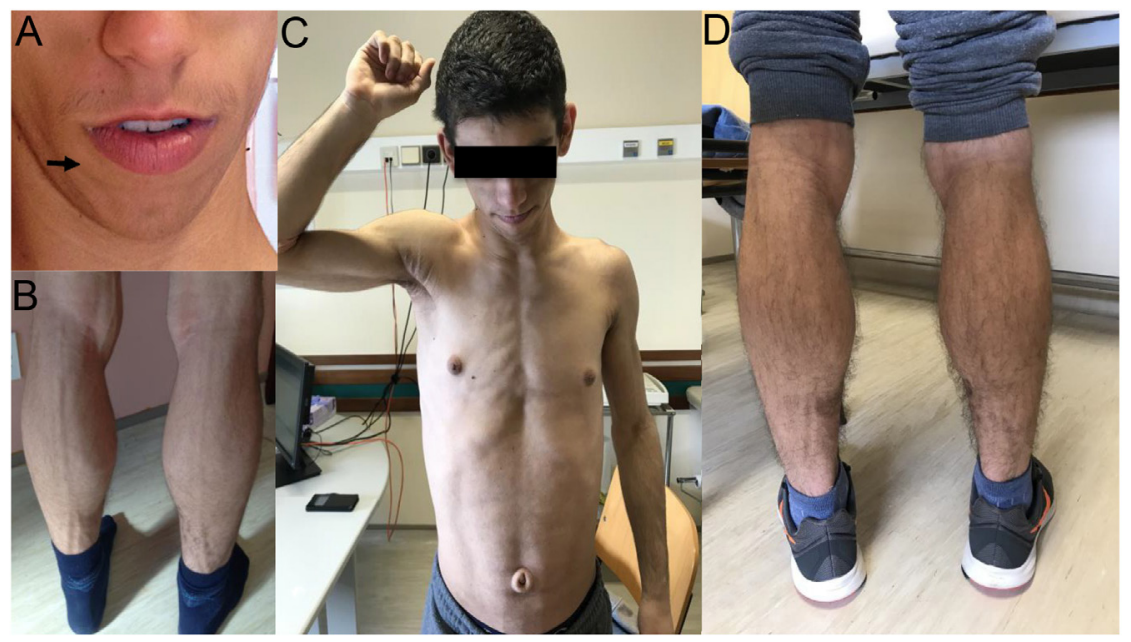

Figure 1

(A) Lipoatrophy of the face, with acromegaloid features. Black arrow shows preservation of fat tissue around the mouth; (B) Prominent muscular hypertrophy and subcutaneous veins; (C) Athletic morphotype, exuberant acanthosis nigricans particularly in the axillae, and umbilical hernia; (D) After 5 years of pioglitazone treatment subcutaneous veins were no longer prominent and there was evidence of subcutaneous adipose tissue. 


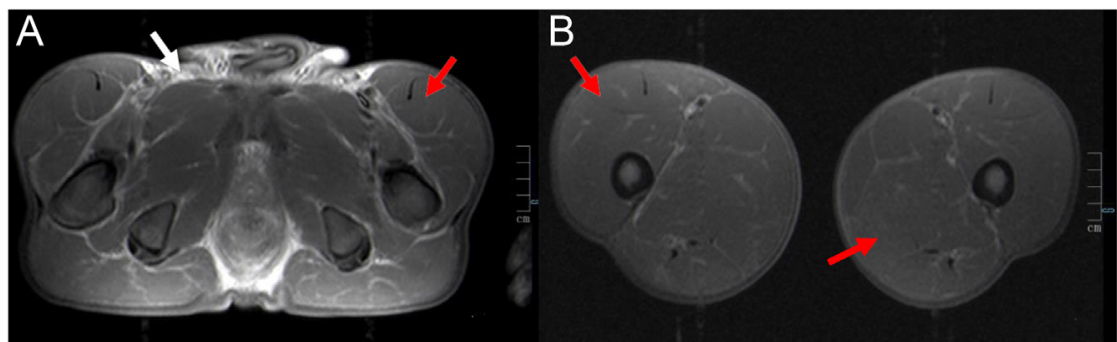

Figure 2

Axial T1-weighted MRI of (A) pelvis and (B) lower limbs, showing shortage of epifascial and intermuscular fat plans (red arrows) and the presence of subcutaneous adipose tissue (white arrow).
$1000 \mathrm{mg}$ twice daily due to diabetes. Although an initial compensation was achieved, secondary failure was documented a few months later with HbA1c rising above $7.5 \%$. For this reason, we obtained the approval status of pioglitazone for the management of diabetes in children under 18 years of age, and we added pioglitazone $15 \mathrm{mg} /$ day twice daily.

\section{Outcome and follow-up}

Since the patient started pioglitazone, and during the last 5 years, the patient $\mathrm{HbA} 1 \mathrm{c}$ has been $6.5 \%$. The $\mathrm{HOMA}_{\mathrm{IR}}$ index decreased to $4.6,11$ months after the patient started pioglitazone. An echocardiogram was performed, showing no abnormality in heart. At 14 years old, despite maintaining good metabolic control, the patient repeated the abdominal ultrasound evaluation. The liver was still enlarged, with increased echogenicity, suggesting steatosis. Due to this finding, the hepatic fat content was estimated by transient elastography (FibroScan ${ }^{\circledR}$, TE) with the determination of controlled attenuation parameter (CAP). This is a reliable and highly reproducible noninvasive method to the diagnosis of hepatic steatosis (7). CAP was $252 \mathrm{~dB} / \mathrm{m}$, a value above the upper limit of normal of $240 \mathrm{~dB} / \mathrm{m}$. To further document the patient's absence of peripheral adipose tissue, an abdominal MRI and a whole body dual-energy X-ray absorptiometry (DEXA), were performed. Total body fat was 6.7\% (normal $18-22 \%$ ), reflecting a severe decrease in peripheral adipose tissue. However, the MRI documented the existence of subcutaneous fat in lower limbs (Fig. 2; abdominal region not scanned due to claustrophobia). Although thiazolidinediones (TZDs) are not the first-line therapy in BSCL (4), our patient has been kept in a good metabolic control with pioglitazone, indicating that this drug may be an alternative of choice in these patients.

\section{Discussion}

A mutation in BSCL2 was the underlying genetic defect identified in our patient. This gene encodes a protein called seipin, which seems to be crucial in adipocyte differentiation. Although lipodystrophy was evident in most of the patient's body, there was the preservation of adipose tissue around the mouth, tongue and palmar face of hands (Fig. 1). To justify this preservation of adipose tissue, some authors hypothesize the existence of two types of fat: a metabolically active type, which is responsible for storing and releasing energy; and another type that has a mechanical function, protecting against shock and friction. According to this theory, these patients have a deficit of metabolically active fat, which justifies the early appearance of complications (8). Thus, both diet and exercise are essential to prevent ectopic fat storage and disease progression (3). Fibrates and omega-3 fatty acids are useful adjunctive therapies in severe hypertriglyceridemia. Regarding diabetes and insulin resistance, metformin is the first-line treatment, because of its ability to increase insulin sensitivity and decrease hepatic gluconeogenesis (4). When metformin titrated to the maximum dose is no longer efficient, insulin in high doses are usually needed. The TZDs were introduced to treat type 2 diabetes because they show a high-affinity to the peroxisome proliferatoractivated receptor-gamma (PPARG) molecule that is able to increase systemic insulin sensitivity. They are also able to partially restore the defective adipogenic process associated with seipin deficiency, providing evidence for the major role of this protein in adipocyte differentiation (9) and their utility in BSCL. They were successfully used to treat HIV-associated and partial lipodystrophies, although very little data about its use in BSCL is available (10). To our knowledge, this is the first report of a patient with BSCL2 mutation with long-term successful treatment with pioglitazone. Arioglu et al. in 2000 were the first to report a 6-month trial of troglitazone in a patient with BSCL (2). The results were surprising, with amelioration of metabolic control and improvement in subcutaneous adipose tissue, suggesting that there may be a transition from the fat stored in the liver to the subcutaneous tissue. Later on, in 2010, Victoria et al. published a very interesting article about a patient with BSCL treated for 1 year with rosiglitazone (10). An increase in leptin levels 
was demonstrated, but after drug discontinuation the leptin levels dropped again. In an in vivo study realized by Prieur and colleagues (9), BSCL2-/- mice were fed with a diet supplemented with pioglitazone for 9 weeks, and the results revealed a threefold increase in weight of residual mesenteric and inguinal fat pads. The authors suggested that the benefit of TZDs in BSCL was associated with the activation of adipose PPARG in the residual fat depots. This evidence indicates that pioglitazone is potentially useful in patients who maintain some active fat deposits, as revealed in MRI scan of our patient's limbs (Fig 2B). The progressive increase in the subcutaneous adipose tissue attenuated the prominence of the veins particularly in the legs (Fig 1B and D). Together with leptin analogs, TZDs are the only drugs capable of inducing the proliferation of subcutaneous adipocytes. While metabolic complications may be easily treated in the early stages of the disease, they are no longer reversible once fully established. At this stage, only leptin analogs are able to control patients, since it acts on pathophysiologic and not in complications mechanisms. As this is a rare disease, it may lead to a delay in diagnosis or misdiagnosis, with the development of early complications and increased mortality at young age. Leptin analogs are a promising therapy but the costs still very high and adverse effects are considerable. Therefore, we judge relevant to testimony our experience, in order to increase the awareness of health care professionals to other therapeutic options.

\section{Declaration of interest}

The authors declare that there is no conflict of interest that could be perceived as prejudicing the impartiality of the research reported.

\section{Funding}

This research did not receive any specific grant from any funding agency in the public, commercial or not-for-profit sector.

\section{Patient consent}

Informed consent was obtained from the parents of the patient included in this paper.

\section{Author contribution statement}

Carolina Chaves wrote the case report and performed literature search for writing discussion with references, João Anselmo helped in writing the case report, reviewed the case report, provided the patient's images, and authorised the final version of the case report, Mariana Chaves was the radiologist responsible for capturing the images, gave an expert opinion, and authorised the final version of the case report, Rui César authorised the final version of the case report. All the authors have accepted responsibility for the entire content of this submitted manuscript and approved submission.

\section{References}

1 Medeiros LB, Dantas VK, Sarmento AS, Agnez-Lima LF, Meireles AL, Nobre TT, Lima J \& Campos J. High prevalence of Berardinelli-Seip congenital lipodystrophy in Rio Grande do Norte State, Northeast Brazil. Diabetolology and Metabolic Syndrome 20179 1-6. (https://doi. org/10.1186/s13098-017-0280-7)

2 Arioglu E, Duncan-Morin J, Sebring N, Rother KI, Gottlieb N, Lieberman J, Herlon D, Kleiner DE, Reynolds J, Premkumar A, et al. Efficacy and safety of troglitazone in the treatment of lipodystrophy syndromes. Annals of Internal Medicine $2000 \mathbf{1 3 3}$ 263-274. (https://doi.org/10.7326/0003-4819-133-4-20000815000009)

3 Araújo-Vilar D \& Santini F. Diagnosis and treatment of lipodystrophy: a step-by-step approach. Journal of Endocrinological Investigation 2019 42 61-73. (https://doi.org/10.1007/s40618-018-0887-z)

4 Brown RJ, Araujo-Vilar D, Cheung PT, Dunger D, Garg A, Jack M, Mungai L, Oral EA, Patni N, Rother KI, et al. The diagnosis and management of lipodystrophy syndromes: a multi-society practice guideline. Journal of Clinical Endocrinology and Metabolism 2016101 4500-4511. (https://doi.org/10.1210/jc.2016-2466)

5 Gomes KB, Pardini VC \& Fernandes AP. Clinical and molecular aspects of Berardinelli-Seip Congenital Lipodystrophy (BSCL). Clinica Chimica Acta: International Journal of Clinical Chemistry 2009402 1-6. (https://doi.org/10.1016/j.cca.2008.12.032)

6 Fraquelli M, Rigamonti C, Casazza G, Conte D, Donato MF, Ronchi G $\&$ Colombo M. Reproducibility of transient elastography in the evaluation of liver fibrosis in patients with chronic liver disease. Gut 200756 968-973. (https://doi.org/10.1136/gut.2006.111302)

7 Magré J, Delépine M, Khallouf E, Gedde-Dahl T, Van Maldergem L, Sobel E, Papp J, Meier M, Mégarbané A, Bachy A, et al. Identification of the gene altered in Berardinelli-Seip congenital lipodystrophy on chromosome 11q13. Nature Genetics 200128 365-370. (https://doi. org/10.1038/ng585)

8 Agarwal AK, Simha V, Oral EA, Moran SA, Gorden P, O'Rahilly S, Zaidi Z, Gurakan F, Arslanian SA, Klar A, et al. Phenotypic and genetic heterogeneity in congenital generalized lipodystrophy. Journal of Clinical Endocrinology and Metabolism 200388 4840-4847. (https://doi.org/10.1210/jc.2003-030855)

9 Prieur X, Dollet L, Takahashi M, Nemani M, Pillot B, Le May C, Mounier C, Takigawa-Imamura H, Zelenika D, Matsuda F, et al. Thiazolidinediones partially reverse the metabolic disturbances observed in Bscl2/seipin-deficient mice. Diabetologia 201356 1813-1825. (https://doi.org/10.1007/s00125-013-2926-9)

10 Victoria B, Cabezas-Agrícola JM, González-Méndez B, Lattanzi G Del Coco R, Loidi L, Barreio F, Calvo C, Lado-Abeal J \& AraújoVilar D. Reduced adipogenic gene expression in fibroblasts from a patient with type 2 congenital generalized lipodystrophy. Diabetic Medicine 201027 1178-1187. (https://doi.org/10.1111/j.14645491.2010.03052.x

Received in final form 27 December 2020

Accepted 23 March 2021 